Accepted manuscript published in:

Altanian, Melanie (Hrsg.) Der Genozid an den ArmenierInnen: Beiträge zur wissenschaftlichen Aufarbeitung eines historischen Verbrechens gegen die Menschlichkeit. Wiesbaden: Springer VS, 2018. https://doi.org/10.1007/978-3-658-20453-2_6

\title{
Menschenwürdeverletzung der Nachfahren durch Genozidleugnung
}

\section{Melanie Altanian}

Im Zusammenhang mit der Aufarbeitung historischer Genozide, wie dem Genozid an den Armeniern, lassen sich auf der Opferseite die folgenden Adressaten unterscheiden: Die direkten Genozidopfer, die Nachfahren der Überlebenden oder indirekten Genozidopfer, sowie die Opfergruppe als solche - also die Kollektivität, respektive soziale Gruppe der „Armenier“. Jede dieser Gruppen kann spezifische Rechte oder Ansprüche an die Aufarbeitung des Verbrechens geltend machen, wobei ich im Folgenden insbesondere auf letztere fokussieren möchte und damit auf die intergenerationelle, kollektive Betroffenheit der Opfergruppe. ${ }^{1}$ Die genozidale Absicht schlägt sich nämlich nicht lediglich in der inhumanen Behandlung von Individuen mit entsprechender Gruppenzugehörigkeit nieder, sondern beinhaltet nach völkerrechtlicher Definition die gewollte Vernichtung der Gruppe als solche und dadurch eine Aberkennung ihrer Legitimität. Dadurch hat die ganze Gruppe einen Grund, sich bedroht und unsicher zu fühlen, auch wenn nicht jedes einzelne Gruppenmitglied physisch oder materiell direkt verletzt wird. Ziel des vorliegenden Beitrags ist es nun, zu zeigen, inwiefern die Opfergruppe durch die offizielle Leugnung des Genozids einer erneuten kollektiven Demütigung zum Opfer fällt. Die Genozidleugnung soll als eigenständige Ungerechtigkeit identifiziert werden, wodurch nicht nur eine gerechte Aufarbeitung des Verbrechens verhindert wird. Vielmehr stellt Genozidleugnung eine erneute Unterminierung der Selbstachtung und damit Würdeverletzung der Betroffenen dar, was sie somit als intrinsisch ethisch verwerflich ausweist. Hierfür soll zunächst die spezifische Grausamkeit von Genozid normativ begründet und die intergenerationelle Natur des Verbrechens aufgezeigt werden. Dies beinhaltet nicht nur eine Begründung der normativen Relevanz von Gruppenzugehörigkeit, sondern auch eine Klärung dessen, welche Rolle das Wissen um sowie die Erinnerung an den Genozid im kulturellen Gedächtnis der Opfergruppe gerechtfertigterweise einnimmt. In einem letzten Schritt wird

\footnotetext{
${ }^{1}$ Der Einfachheit halber werde ich von „Opfer“- bzw. „Tätergruppe“ sprechen, obschon ich damit nicht implizieren möchte, dass Nachfahren notwendigerweise den „Opfer“- bzw. „Täterstatus“ erben. Als Armenierin dritter Generation wäre es unplausibel, zu behaupten, ich sei Opfer des Genozids, genauso wie es unplausibel wäre, zu behaupten, dass die Bevölkerung der heutigen Türkei Schuld am Genozid sei. Dies ist jedoch zu unterscheiden von der Bedeutung, die der Genozid gerechtfertigter Weise für Nachfahren der Opfer hat, sowie der gesellschaftlichen und staatlichen Verantwortung, den Genozid aufzuarbeiten.
} 
begründet, wie unter diesen Bedingungen Genozidleugnung eine spezifische, epistemische Ungerechtigkeit gegenüber Nachfahren darstellt, indem ihnen die Glaubwürdigkeit und damit epistemische Autorität auf der Grundlage ihrer sozialen Zugehörigkeit abgesprochen wird, wodurch sie erneut gedemütigt werden.

\section{Würde, Selbstachtung und Demütigung}

Die Würde gilt als beständig und unveräußerlich. Dahinter steckt die Idee, dass Menschen Würde besitzen und diese lediglich verletzt werden kann. Der inhärente Wert des Menschen verschwindet nicht dadurch, dass es für ihn unmöglich wird, ein würdevolles Leben zu führen, denn nichtsdestotrotz bleibt er ein Mensch. Wozu es aber führen kann ist, dass ein Mensch seine Selbstachtung verliert: Wer von seinen Mitmenschen nicht mehr als Mensch geachtet wird, der läuft Gefahr, sich selbst nicht mehr als Mensch achten zu können. Avishai Margalit versteht unter Selbstachtung eine Haltung, die wir uns selbst gegenüber als Subjekte mit inhärentem Wert entgegenbringen sollten. Die Würde ist dabei die externe Dimension der Selbstachtung bzw. Ausdruck dieser Selbstachtung, so wie Stolz der Ausdruck unseres Selbstwertgefühls ist. ${ }^{2}$ Man kann jedoch Selbstachtung haben ohne Würde zu besitzen, also ohne diese Selbstachtung positiv zu bekunden. Wer aber in seiner Selbstachtung verletzt wird, der ist auch in seiner Würde verletzt. Die Möglichkeit einer Verletzung menschlicher Selbstachtung ist wiederum abhängig von bestimmten menschlichen Eigenschaften und Umständen, welche objektive normative Gründe liefern, wann und weshalb sich jemand legitimerweise in seiner Selbstachtung verletzt erachten kann. Ob eine Verletzung der Selbstachtung vorliegt ist also nicht abhängig von persönlichen Befindlichkeiten, wie es bei einer Verletzung des Selbstwertgefühls der Fall ist. Wie ich im Folgenden darlegen werde, liegen objektive normative Gründe für die Verletzung der Selbstachtung hingegen dann vor, wenn man gedemütigt wird.

Demütigung ist laut Margalit eine Grausamkeit, die der Tatsache geschuldet ist, dass wir aufgrund von Handlungen symbolischer Natur leiden können. Sie gehen zwar meist einher mit physischen Schädigungen, doch es ist insbesondere die Eigenschaft, symbolisches Leid empfinden zu können, welche Nicht-Demütigung rechtfertigt. ${ }^{3}$ Das spezifische symbolische Element, welches für unsere Überlegungen relevant ist, findet sich im Anspruch des Täters auf existentielle Überlegenheit, wodurch er dem Opfer übermittelt, dass es nicht mehr länger als Mitglied der Menschengemeinschaft anerkannt wird. Dieser Anspruch ist insofern symbolisch,

\footnotetext{
${ }^{2}$ Vgl. Margalit 2012, S. 61

${ }^{3}$ Ebd., S. 84. Dies entspricht im Grunde der Unterscheidung zwischen physischem und psychischem Leid.
} 
als das Opfer offensichtlich immer noch ein Mensch ist und auch bleibt. Margalit liefert nun keine Definition des Begriffs der Demütigung, sondern nennt drei Sinnebenen von Demütigung und damit eine phänomenologische Beschreibung ihrer Mechanismen:

(i) Menschen so zu behandeln, als ob sie keine Menschen wären;

(ii) der Ausschluss von Menschen aus der Menschengemeinschaft und

(iii) Handlungen, die zum Verlust der Selbstkontrolle führen oder diesen verdeutlichen.

Diese Elemente sind jeweils hinreichend, aber nicht zusammen notwendig für Demütigung, denn sie sind miteinander verknüpfte Phänomene desselben Begriffs. Unsere Selbstachtung aufrecht erhalten zu können heisst also auch, Selbstkontrolle zu bewahren, respektive uns dem Willen anderer Menschen nicht unterwerfen zu müssen. ${ }^{4}$ Daher geht mit Demütigung immer auch ein Verlust der Selbstkontrolle einher. Die Idee der Selbstkontrolle ist wichtig, da es ansonsten nicht klar wäre, weshalb andere Leute eine Haltung beeinflussen können, welche wir uns selbst gegenüber einnehmen sollten. Die Macht der anderen besteht gerade darin, dass sie uns aus der Menschengemeinschaft ausschliessen und somit eine existenzielle Bedrohung für uns darstellen können. Der Gedemütigte ist den Interessen seines Peinigers hilflos ausgeliefert und dies „manifestiert sich in der Angst, nicht mehr für die eigenen lebenswichtigen Interessen sorgen zu können“. ${ }^{5}$ Dabei muss betont werden, dass es Margalit um institutionelle Demütigung geht, wo es klarerweise schwieriger wird, die Kontrolle über unsere existentiellen Interessen zu wahren - denn Zweck sozialer Institutionen ist es gerade, unter Berücksichtigung der fundamentalen Interessen der Mitglieder einer Gesellschaft das menschliche Handeln zu organisieren und strukturieren. Das Ausmass, respektive Machtverhältnis ist bei der Demütigung also entscheidend. Je mehr Macht eine Institution über die Lebensverhältnisse der ihr unterstehenden Individuen hat, das heisst, je wichtiger die Rolle dieser Institutionen in der Erfüllung ihrer Lebensbedingungen, desto grösser ist die Gefahr der Demütigung. Demütigung stellt also eine ultimative Bedrohung der menschlichen Existenz dar, was für Genozid besonders kennzeichnend ist und sich auch in entsprechender offizieller Rhetorik seitens der Täter niederschlägt, die den Anspruch auf existentielle Überlegenheit reklamieren. So wurden im Osmanischen Reich die „ungläubigen Armenier“ beispielsweise als Mikroben und Parasiten

\footnotetext{
${ }^{4}$ Ebd., S. 150

${ }^{5}$ Ebd., S. 127. Wir können unsere Selbstkontrolle auch durch Naturkatastrophen oder Unfälle verlieren und dadurch traumatisiert sein. Jedoch stellt die Demütigung ein Kontrollverlust dar, der uns spezifisch in unserer sozialen Lebenssphäre betrifft. Es erschüttert das lebensnotwendige Grundvertrauen in unsere Selbstverfügbarkeit und Handlungsfähigkeit im Zusammenleben mit andern Menschen. Das durch Demütigung erfahrene Trauma scheint also eine andere Qualität zu haben und erfordert daher auch andere Massnahmen der Ver- bzw. Aufarbeitung: „[...] understanding humanly caused trauma requires broadly based political movements that resist the suppression of survivor accounts, and politically committed witnesses who encourage and acknowledge narratives of harm.“ (Campbell 2003, S. 71, zit. n. Herman 1992).
} 
bezeichnet, als Schädlinge, die es zu bekämpfen und bis zuletzt auszumerzen gilt. ${ }^{6}$ Diese Mechanismen der Dehumanisierung führen zum Ausschluss der Armenier aus der Menschheitsfamilie, wodurch man nicht mehr dazu angehalten ist, sie als Menschen zu achten und entsprechend zu behandeln. ${ }^{7}$ Ein Verweis auf den Vorwurf der Ungläubigkeit ist in diesem Zusammenhang relevant, denn dies begründet mitunter auch, weshalb andere christliche Bevölkerungsgruppen dem Genozid zum Opfer fielen. Dazu gehörte auch, dass diejenigen vornehmlich Frauen und Kinder, jedoch auch einige handwerklich „nützliche“ Armenier -, die am Leben gelassen wurden, zum Islam zu konvertieren hatten oder dies durch Zwangsheiraten bzw. durch das anschliessende Aufwachsen von Waisenkindern in türkischen Waisenhäusern oder Haushalten notwendigerweise geschah. ${ }^{8}$

Genozid ist nicht das einzige Verbrechen, welches durch Demütigung gekennzeichnet ist. Es ist jedoch ein paradigmatisches Beispiel dafür, insbesondere in Form des Ausschlusses einer bestimmten moralisch legitimen identitätsstiftenden Gruppe. Im Folgenden werde ich genauer auf das Konzept der Demütigung im Zusammenhang mit der Zugehörigkeit zu legitimen identitätsstiftenden Gruppen eingehen und damit aufzeigen, inwiefern Genozid als spezifisches Verbrechen gegen die Menschlichkeit verstanden werden kann.

\section{«Die Gruppe als solche»: Genozid als spezifisches Verbrechen gegen die Menschlichkeit} Erst einige Jahre nach dem Ende des zweiten Weltkriegs, respektive des Holocausts wurde das Verbrechen des Genozids als neuer Tatbestand und „besonderes“ Verbrechen gegen die Menschlichkeit im internationalen Völkerstrafrecht verabschiedet. ${ }^{9}$ Die teilweise unklare oder unplausible Definition der UN Konvention ist Resultat eines politischen Konsenses, was bis heute zu einer regen Debatte und Kritik an ihr geführt hat. Unter anderem wird bemängelt, dass sie lediglich die Zerstörung ethnischer, religiöser, rassischer und nationaler Gruppen berücksichtigt, wodurch andere soziale oder politische Gruppen ausgeschlossen werden.

\footnotetext{
${ }^{6}$ Vgl. Livingstone Smith 2011, S. 144, zit. n. Dadrian 1996, S. 159.

${ }^{7}$ Die praktischen Konsequenzen der Dehumanisierung - nämlich der tatsächliche Ausschluss und die Vernichtung der Gruppe - werden nicht nur institutionell legitimiert, sondern auch befördert.

${ }^{8}$ Zur jungtürkischen Assimilierungspolitik siehe den Beitrag von Hilmar Kaiser in diesem Bd. Konversion war demnach nur wenigen als Alternative zur Deportation bzw. Ermordung vorbehalten, abhängig von den regionalen Gegebenheiten und wirtschaftlichen Interessen oder Notwendigkeiten. Ausserdem durften diese jeweils nicht mehr als 5\% der regionalen muslimischen Bevölkerung ausmachen - eine Bedingung, die sich angesichts der totalen Vernichtung oder Vertreibung der dort ansässigen armenischen Bevölkerung meist erübrigte. Damit hing ausserdem zusammen, dass die Regierung Massnahmen ergreifen musste, um in der Lage zu sein, konvertierte Armenier von „,richtigen Muslimen“ zu unterscheiden, was ein weiteres Indiz inhärenter Diskriminierung darstellt und diese institutionell beförderte.

${ }^{9}$ Ich spreche hier und im Folgenden von einem „besonderen“ Verbrechen insofern, als es mir lediglich darum geht, Genozid von anderen Massenverbrechen im Rahmen von Verbrechen gegen die Menschlichkeit qualitativ zu unterscheiden. Ich möchte dadurch jedoch noch nicht die Annahme vertreten, dass Genozid moralisch als das „grösste“ oder „schlimmste“ aller Verbrechen zu bewerten sei, auch wenn eine solche Bewertung für die Justiz bzw. in Hinblick auf das Strafmass von Bedeutung sein mag.
} 
Versucht man jedoch, die Schutzwürdigkeit dieser vier Gruppen normativ zu begründen - wie ich es später ausführen werde - ist nicht evident, weshalb sich Genozid in der beabsichtigten Zerstörung „bloss“ dieser Gruppen niederschlagen kann. Die Hintergrundüberlegungen, die Raphael Lemkin zur Initiierung eines neuen völkerstrafrechtlichen Tatbestandes führten, sahen jedenfalls eine breitere Definition vor. So spricht Lemkin bereits 1933 von „acts of extermination directed against the ethnic, religious or social collectivities whatever the motive (political, religious, etc.); for example massacres, pogroms, actions undertaken to ruin the economic existence of the members of a collectivity, etc.” (eigene Hervorhebung). ${ }^{10}$

Auch unter Genozidwissenschaftlern wird gelegentlich ein erweitertes Verständnis schützenswerter Gruppen anerkannt. ${ }^{11}$ Larry May schlägt beispielsweise vor, dass der Wortlaut im internationalen Recht zu ,,a publicly recognized group that is relatively stable and significant for the identity of its members, such as a national, ethnical, racial or religious group” geändert werden soll. ${ }^{12}$ So hat zumindest die Schweiz seit 2010 soziale wie politische Gruppen in ihrem Bundesgesetz integriert. ${ }^{13}$ Doch abgesehen von der Frage danach, welche Gruppen als schützenswert gelten sollen, besteht ein zentrales Merkmal des Verbrechens in der beabsichtigten Zerstörung derselben. Die Absicht, eine Gruppe zu zerstören, muss nicht unbedingt in der Tötung all ihrer Mitglieder - oder eines Teils davon - resultieren. Andere Handlungen, die dem Tatbestand des Genozids gemäss der Konvention genügen, sind, wenn aus dieser Absicht heraus bei Mitgliedern dieser Gruppe schwere körperliche und seelische Schäden verursacht werden; ihnen vorsätzlich Lebensbedingungen auferlegt werden, die geeignet sind, ihre körperliche Zerstörung ganz oder teilweise herbeizuführen; die Verhängung von Maßnahmen, die auf die Geburtenverhinderung innerhalb der Gruppe gerichtet sind; sowie die gewaltsame Überführung von Kindern der Gruppe in eine andere Gruppe. ${ }^{14}$ Unter körperlichen und seelischen Schäden können meines Erachtens auch die Angstzustände verstanden werden, wodurch Mitglieder gezwungenermassen jegliche Gruppenmerkmale

\footnotetext{
${ }^{10}$ Raphael Lemkin, Acts Constituting a General (Transnational) Danger Considered as Offences Against the Law of Nations, Additional explications to the Special Report presented to the $5^{\text {th }}$ Conference for the Unification of Penal Law in Madrid (14-20 October 1933), verfügbar unter: http://www.preventgenocide.org/lemkin/madrid1933-english.htm. [Zugegriffen: 03. Mai 2016]

${ }^{11}$ Bsp. Chalk 1994, S. 47ff.; Charny 1994, S. 64ff.; Bauer 2009, S. 25; May 2010, S. 58; sowie Revised and updated report on the question of the prevention and punishment of the crime of genocide (Whitaker Report), UN Doc. E/CN.4/Sub.2/1985/6, 2 July 1985

12 May 2010, S. 57, eigene Hervorhebung.

${ }^{13}$ Vgl. Bundesgesetz über die Änderung von Bundesgesetzen zur Umsetzung des Römer Statuts des Internationalen Strafgerichtshofes, verfügbar unter: https://www.admin.ch/opc/de/officialcompilation/2010/4963.pdf

${ }^{14}$ Vgl. Art. 2, Übereinkommen vom 9. Dezember 1948 über die Verhütung und Bestrafung des Völkermordes, offizielle Schweizer Übersetzung des französischen Originaltexts. Verfügbar unter: https://www.admin.ch/opc/de/classified-compilation/19994549/201406110000/0.311.11.pdf
} 
ablegen oder verdrängen, sich also aus Überlebensgründen assimilieren, wobei dies aus sozialen Gegebenheiten resultiert und nicht weil beispielsweise eine bestimmte Lebensweise nicht mehr mit den natürlich vorhandenen Ressourcen aufrechterhalten werden kann. Vom Wortlaut her ist Genozid also das einzige Verbrechen gegen die Menschlichkeit, welches den Schaden an einer sozialen Gruppe primär berücksichtigt und nicht die Schädigung bestimmter Individuen aufgrund ihrer Gruppenzugehörigkeit, wie es beim Verbrechen der Verfolgung auch ist. Hierfür ist es nun notwendig, zu begründen, was diese Gruppen normativ wertvoll macht, sodass ihre Zerstörung ein „besonderes“ Verbrechen gegen die Menschlichkeit darstellt. So spielt es für die Welt insgesamt keine Rolle, wie viele verschiedene Sprachen die Menschen kreieren und aufrechterhalten wollen, oder wie viele unterschiedliche Religionen und Kulturen sich aus ihnen bilden. Der Zustand der Welt als eine bessere oder schlechtere Welt ist also nicht abhängig von der Anzahl sozialer Gruppen. ${ }^{15}$ Eine mögliche Begründung des intrinsischen Werts sozialer Gruppen besteht darin, dass sie die intrinsische Struktur wichtiger menschlicher Beziehungen haben. Die Zerstörung einer Gruppe bedeutet also die Zerstörung besonderer Beziehungen zwischen Individuen, welche die Gruppe konstituieren. ${ }^{16}$ Die Wichtigkeit dieser Beziehungen für die Gruppenmitglieder bedeutet, dass sie ein gemeinsames Interesse daran haben, dass die Gruppe (weiter-)besteht. Da ihr Selbstverständnis teilweise auf der Gruppenzugehörigkeit beruht - ob selbst gewählt oder aufgrund externer, aufgedrängter Umstände - hat die Existenz der Gruppe intrinsischen Wert für ihre Mitglieder, jedoch nicht unabhängig von ihnen. Doch worin besteht der Wert dieser Beziehungen, beziehungsweise wie hängt dies mit unserem Selbstverständnis zusammen?

Gemäss Avishai Margalit und Joseph Raz ist es mitunter die Tatsache, dass wir identitätsstiftenden Gruppen zugehören, was uns empfänglich für Demütigung macht. Diese Form der Demütigung durch den Ausschluss einer moralisch legitimen identitätsstiftenden Gruppe - und somit einer spezifischen Art und Weise, wie sich die Mitglieder als Menschen (viz. Personen) zum Ausdruck bringen - wird uns helfen, zu verstehen, welche relevanten normativen Überlegungen in der Beziehung zwischen Gruppenzugehörigkeit und Identität im Spiel sind. Da es sich bei der Demütigung außerdem um ein normatives Konzept handelt, beschränkt sich dessen Anwendbarkeit auf moralisch legitime identitätsstiftende Gruppen. ${ }^{17}$

\footnotetext{
${ }^{15}$ Vgl. May 2010, S. 66f.

${ }^{16}$ Es ist Gegenstand einer intensiven philosophischen Debatte, in welcher Art von „besonderer Beziehung“ Menschen zueinanderstehen sollten, bzw. welche Bedingungen dabei erfüllt sein müssen, um soziale Gruppen zu konstituieren. Darauf werde ich hier aber nicht weiter eingehen.

${ }^{17}$ Auch die Mafia kann identitätsstiftend sein. Doch Mitglieder der Mafia haben keinen gerechtfertigten Grund, sich als gedemütigt zu erachten, wenn der Staat ihnen verbietet, die Mafiakultur auszuleben bzw. aufrecht zu erhalten, denn die Mafia ist eine Gruppe, welche ihre eigenen Mitglieder demütigt. Vgl. Margalit 2012, S. 145 und 265f. Damit betont Margalit, dass es sowohl legitime als auch illegitime Aspekte einer persönlichen Identität
} 
Doch inwiefern kann der Gruppe eine Selbstachtung zugesprochen werden, wodurch sie Opfer der Demütigung werden kann? Bei Margalit operiert dies hauptsächlich über die Idee einer durchdringenden Kultur, welche letztlich ein Recht auf kulturelle Zugehörigkeit begründet. Eine identitätsstiftende Gruppe ist demnach gekennzeichnet durch eine Kultur, die verschiedene wichtige Lebensbereiche der Mitglieder durchdringt. Das heißt, sie formt größtenteils die Vorlieben sowie Möglichkeiten der Mitglieder und versorgt sie mit einer sicheren Identität. Die Idee einer sicheren oder stabilen Identitätsgrundlage schließt sich daraus, dass die Zugehörigkeit zu solchen identitätsstiftenden Gruppen keine Leistung ist, die bewertet werden kann. Es untersteht keiner Leistung, identitätsstiftenden Gruppen zuzugehören, sondern die Zugehörigkeit ist durch wechselseitige informelle Anerkennung gegeben, wobei die Kultur intergenerationell durch Sozialisierung weitergegeben wird. ${ }^{18}$ Identitätsstiftende Gruppen legen also die Bandbreite möglicher, verfügbarer Lebensoptionen fest. Daher stellen sie im Grunde unterschiedliche Arten und Weisen dar, Mensch zu sein bzw. sich als Mensch auszudrücken. Jemanden also nicht aufgrund seiner Zugehörigkeit zu einer solchen Gruppe zu demütigen, heißt, den Wert kultureller Zugehörigkeit zu respektieren.

Margalit und Raz geben letztlich keine abschließende Definition identitätsstiftender Gruppen, auch um ihre Ausgestaltung möglichst offen zu halten. Sie haben sich vielmehr darauf konzentriert, diejenigen Merkmale aufzuzeigen, die begründen, weshalb sie für das Selbstverständnis der Mitglieder relevant sind. Denn aufgrund dieser besonderen Wichtigkeit für das Selbstverständnis der Gruppenmitglieder führt die Verspottung, Unterdrückung oder Diskriminierung, also Formen des Ausschlusses solcher Gruppen gemäß Margalit „zu Schmerz, Demütigung, Degradierung, moralischer Herabsetzung und Kränkung; sie liefern auch den sich mit diesen Gruppen identifizierenden Mitgliedern einen rationalen Grund, sich [in ihrer Selbstachtung] verletzt zu fühlen“. ${ }^{19}$ Denn einerseits werden durch diesen Ausschluss die Gruppenmitglieder als Menschen zurückgewiesen, indem die Art und Weise zurückgewiesen wird, in der sie sich als Menschen zum Ausdruck bringen. Andererseits führt ein solcher Ausschluss zu einem Verlust der Kontrolle über ihre Identitätsgrundlage. Damit ist nicht ein Kontrollverlust über Identitätsmerkmale gemeint, denn diese werden ja gerade als

\footnotetext{
geben kann. Dasselbe gilt für Neonazis oder den Daesh, der per definitionem genozidal ist - also nur ihrer eigenen Gruppe ein Existenzrecht zuspricht.

${ }^{18}$ Dies schließt nicht aus, dass solche Sozialisierung fehlschlagen kann, oder sich manche von dieser Sozialisierung bewusst distanzieren möchten. Durch den Fokus auf die Übertragung der Gruppenkultur durch Sozialisierung wird außerdem die historische Natur der Gruppen betont, um die es sich hier handelt. Angesichts dessen, dass sie zumindest teilweise durch eine gemeinsame Kultur konstituiert sind, teilen sie auch eine gemeinsame Geschichte, welche es überhaupt ermöglicht, von einer Entwicklung und Weitergabe von Kultur zu sprechen. Vgl. Margalit und Raz 1990, S. 444f.

${ }^{19}$ Margalit 2012, S. 145
} 
Diskriminierungsgrund herangezogen und unterliegen primär, wie bereits angedeutet, auch nicht der Kontrolle der Gruppenmitglieder (sei es die Hautfarbe, Ethnie, Religion, das Geschlecht o.ä.). Vielmehr geht es um den Verlust der Kontrolle über die eigene Ausdrucksund Handlungsfreiheit, die wir im Rahmen unserer Identität realisieren. Durch Demütigung wird dabei die Identitätsgrundlage als Anlass genommen, diese Freiheiten einzuschränken. ${ }^{20}$ Anhand dieser Überlegungen soll nun genauer darauf eingegangen werden, inwiefern Genozid zu einem generationenübergreifenden Thema wird.

\section{Erinnerung und Selbstachtung: Das Schicksal der Nachfahren}

Um in einem letzten Schritt die Genozidleugnung als besondere epistemische Ungerechtigkeit gegenüber den Nachfahren zu identifizieren, muss zunächst untersucht werden, welchen Stellenwert das Wissen um den Genozid in der Gegenwart für sie hat oder gar haben sollte. Die Aufarbeitung historischer Genozide ist insofern speziell, als die Täter wie auch Opfer größtenteils nicht mehr leben. Nun ist es aber falsch, daraus den Schluss zu ziehen, dass der Anspruch an eine entsprechende Aufarbeitung des Verbrechens dadurch aufgehoben ist. Ich möchte hier nun nicht genauer darauf eingehen, inwiefern sich eine kollektive Verantwortung seitens der Nachfahren der „Tätergruppe“ begründen lässt, sondern zunächst die legitimen Ansprüche seitens der Nachfahren der „Opfergruppe“ untersuchen. Es gilt im Hinblick auf die Nachfahren der „Tätergruppe“ jedoch zu betonen, dass es sich nicht primär um eine Schuldübernahme für das Verbrechen handelt, sondern um die Verantwortung darüber, wie mit den Konsequenzen des Verbrechens umgegangen werden soll. Aus der moralischen, respektive sozialgesellschaftlichen Perspektive sollte die Wiederherstellung der Gerechtigkeit über juristische Bestrafung, i.e. punitive Gerechtigkeit und Reparationszahlungen und somit materielle Wiedergutmachung hinausgehen, insbesondere, wenn es sich um ein Verbrechen wie Genozid handelt. Eine solche Herangehensweise wird auch unter dem Paradigma der „restaurativen Gerechtigkeit“ vertreten, welche eine Konflikttransformation durch gemeinschaftliche bzw. soziale Aspekte wie Anerkennung und Erinnerung vermehrt in den Vordergrund rückt. ${ }^{21}$ Genozid zerstört schliesslich auch die fundamentale Grundlage der sozialen Ordnung, und nicht „bloss“ die rechtliche Ordnung.

\footnotetext{
${ }^{20}$ Vgl. Kohler 2014, S. 8.

${ }^{21}$ Vgl. Walker 2006, S. 384f.; De Greiff 2006, S. 451-77. Damit ist nicht gemeint, dass es mit symbolischen, respektive versöhnlichen Massnahmen getan wäre. Vielmehr stellen letztere eine vorhergehende klare Verurteilung des Verbrechens voraus, was insbesondere in unserem Kontext der Genozidleugnung wichtig ist hervorzuheben.
} 
Ein essentieller Bestandteil dieser gesamtgesellschaftlichen Aufarbeitung wird juristisch als das „Recht auf Wahrheit“ bezeichnet. ${ }^{22}$ So haben Überlebende und Nachfahren beispielsweise das Recht, zu erfahren, was mit ihren Angehörigen passiert ist, um den Verlust entsprechend betrauern und für sich verarbeiten zu können. ${ }^{23}$ Das Recht auf Erinnerung und ihre Bewahrung hängt mit dem Recht auf Wahrheit zusammen, denn sie ermöglicht unter anderem die Vermittlung und Weitergabe von Wahrheit. Dabei ist „Wahrheit“ hier nicht im philosophisch bzw. epistemologisch umstrittenen Sinne gemeint, sondern meint im Kontext der Vergangenheitsarbeit schlicht das Wissen darüber, was passiert ist, respektive eine Gewissheit über die historischen Tatsachen, die für die Bestimmung eines erfahrenen Unrechts relevant sind, sowie deren angemessene Bewertung als Unrecht. Erinnerung ist aber nicht nur instrumentell wertvoll, indem dadurch normativ relevantes Wissen vermittelt und weitergegeben werden kann. Im Zusammenhang mit der Erinnerung an Verbrechen, wie dem Verbrechen des Genozids, wird Erinnerung zu einer intrinsisch moralisch wertvollen Aktivität, indem sie moralische Werte befördert. Diese Sicht formuliert Jeffrey Blustein im Rahmen eines ethischen Expressivismus: eine bestimmte Theorie rationaler Handlung, sowie daraus abgeleitet, eine Werttheorie. Eine Theorie rationaler Handlung insofern, als sie in adäquater Weise unsere rationale Haltung gegenüber Menschen und anderen intrinsisch wertvollen Dingen ausdrückt. ${ }^{24}$ Eine Werttheorie insofern, als Aussagen darüber, was wertvoll ist, Aussagen darüber sind, was es rationalerweise als wertvoll zu erachten gilt. Personen sind demnach wertvoll, weil es rational ist, ihnen gegenüber eine „favorable attitude“ einzunehmen. Angewandt auf unser Beispiel der Erinnerung an Opfer bedeutet dies gemäß Blustein: Ob es wertvoll ist oder nicht, sich des Verbrechens zu erinnern, hängt davon ab, ob diese Erinnerung eine Haltung gegenüber den Opfern ausdrückt, die es rationalerweise einzunehmen gilt. Blustein nennt in diesem Zusammenhang drei intrinsisch wertvolle, rationale Haltungen oder „expressivistische Normen“: Selbstachtung (self-respect), Achtung von Personen (respect for persons) sowie Treue (fidelity) gegenüber den Verstorbenen. ${ }^{25}$ Das Erinnern an Genozid kann somit als Ausdruck der Selbstachtung verstanden werden, genauer ihrer Wiederbehauptung, mit dem Ziel, der Gruppe wieder ein Ansehen innerhalb der politischen Gemeinschaft zu geben,

\footnotetext{
${ }^{22}$ Vgl. UN Human Rights Commission, Updated Set of principles for the protection and promotion of human rights through action to combat impunity, E/CN.4/2005/102/Add.1, S. 7f. Verfügbar unter: https://documentsdds-ny.un.org/doc/UNDOC/GEN/G05/109/00/pdf/G0510900.pdf?OpenElement

${ }^{23}$ Angela Moré weist in ihrem Beitrag des vorliegenden Bands darauf hin, dass durch solche Verbrechen verursachte Traumata unbewusst auch an Nachfolgegenerationen weitergegeben werden können. Wahrheit und Erinnerung werden hier insofern relevant, als sie psychotherapeutische Wichtigkeit haben und der

Traumaverarbeitung dienen.

${ }^{24}$ Blustein 2014, S. 183, zit. n. Anderson 1993, S. 17

${ }^{25}$ Ebd., S. 184
} 
aus welcher sie ungerechterweise ausgeschlossen wurde. ${ }^{26}$ Dies ist notwendig, da durch Genozid, wie bereits erwähnt, eine moralisch legitime Gruppe nicht nur aus der politischen Gemeinschaft, sondern aus der Menschheitsfamilie ausgeschlossen wird und ihren Gruppenmitgliedern dadurch eine existentielle Ablehnung widerfährt. Der moralische Wert der Erinnerung an vergangene Genozide liegt also nicht nur in der Erinnerung an die Toten oder die Opfer generell, sondern auch in der Wiederbehauptung der Legitimität und politischen Wiedereingliederung der Gruppe, weshalb dies auch für gegenwärtig und zukünftig lebende Generationen relevant wird.

Das Erinnern an einen Genozid kann also als symbolische Praxis verstanden werden, wodurch eine intrinsisch moralisch wertvolle Haltung ausgedrückt wird. Während Bedürfnisse nach materieller Wiedergutmachung sicherlich wichtig sind und bei der Vergangenheitsarbeit mitbeachtet werden müssen, gewinnen diese Massnahmen erst dann an Bedeutung und Aufrichtigkeit, wenn sie von Aktivitäten mit symbolischem Gehalt begleitet werden. Umgekehrt sollten symbolische Gesten jedoch auch praktische Folgen haben, das heisst, von materieller Wiedergutmachung begleitet werden, damit ihnen nicht der Vorwurf der „heissen Luft“ gemacht werden kann. Offizielle, politische Entschuldigungen sind nebst der Erinnerung solche symbolischen Praktiken, die auf der sozialen Ebene genauso bedeutsame Haltungen ausdrücken können. Um dies zu erreichen, müssen Entschuldigungen jedoch als ernsthaft und authentisch wahrgenommen werden können, was eine äusserst sensible und komplexe Angelegenheit ist. ${ }^{27}$

Die Erfahrung des Genozids wird dadurch legitimerweise essentieller Bestandteil des kulturellen Gedächtnisses der Gruppe, wodurch das Wissen um den Genozid über Generationen hinweg weitergegeben wird. ${ }^{28}$ Unter diesen Bedingungen können Nachfahren der Opfergruppe durch die Genozidleugnung erneut einer Demütigung zum Opfer fallen, nämlich in Form einer

\footnotetext{
${ }^{26}$ Ebd., S. 188

${ }^{27}$ Vgl. beispielsweise ICTJ Report (2015), More than Words: Apologies as a Form of Reparation, verfügbar unter https://www.ictj.org/sites/default/files/ICTJ-Report-Apologies-2015.pdf. [Zugegriffen: 27. März 2016]. So wurde beispielsweise die vom ehemaligen türkischen Ministerpräsidenten Ahmet Davutoğlu zum 100. Gedenktag des Armeniergenozids geäusserte Mitgefühlsbekundung gegenüber „Kinder und Enkelkinder der Armenier, die ihr Leben bei Deportationen 1915 verloren" von vielen ArmenierInnen als heuchlerisch zurückgewiesen. Das geäusserte Mitgefühl zeige weder eine Anerkennung der Verbrechen, noch ein entsprechendes Verantwortungsbewusstsein seitens der Regierung; es äussere kein Bedauern und biete auch keine Entschädigungen an; siehe http://www.spiegel.de/politik/ausland/tuerkei-ahmet-davutoglu-aeussertmitgefuehl-mit-armeniern-a-1029629.html. [Zugegriffen: 27. März 2016]

${ }^{28}$ Andererseits wird der Ruf nach Erinnerung und Völkermordanerkennung ja gerade besonders in jenen Fällen stark, in denen der Genozid eben offiziell geleugnet wird. Dadurch werden Betroffene stets aufs Neue herausgefordert, sich gegenüber den Leugnenden zu rechtfertigen, bzw. zu beweisen, dass sie im Recht sind.
} 
epistemischen Ungerechtigkeit, wodurch ihnen die Glaubwürdigkeit aufgrund eines Vorurteils gegenüber ihrer sozialen Identität abgesprochen wird.

\section{Demütigung der Nachfahren durch Genozidleugnung}

Wie bereits erläutert wird Demütigung vom Gedemütigten als Kontrollverlust erfahren, respektive zeigt sie sich aus der Perspektive des Demütigenden als Ausschluss von Menschen aus der Menschengemeinschaft. Verbunden mit diesem Mechanismus, Menschen zu behandeln, als ob sie keine Menschen wären - als ob sie Bestien, Parasiten oder Objekte wären - ist ein negatives Vorurteil. Das Vorurteil ist dabei nicht, die Armenier allesamt als Parasiten $\mathrm{zu}$ bezeichnen, was eher metaphorisch gemeint ist. Vielmehr sind es Vorurteile wie die Armenier als feindselig, intrigant, illoyal, unehrlich, etc. zu diskreditieren, was schlimmstenfalls dazu führen kann, sie aus der eigenen Gemeinschaft und im Falle des Genozids auch aus der Menschengemeinschaft auszuschliessen. Negative Vorurteile können seitens der Betroffenen unterschiedliche Rechte infrage stellen, respektive auf unterschiedliche Arten und Weisen ihre Selbstachtung verletzen. Miranda Fricker identifiziert dabei eine spezifisch epistemische Art von Ungerechtigkeit, die aus negativen Vorurteilen resultieren kann. ${ }^{29}$ Das heisst eine Ungerechtigkeit innerhalb unserer sozial situierten Praxis, Wissen zu generieren und weiterzugeben, sowie unsere sozialen Erfahrungen uns selbst und andern gegenüber verständlich zu machen. Im Folgenden möchte ich zeigen, inwiefern Genozidleugnung als eine epistemische Ungerechtigkeit gegenüber den Nachfahren identifiziert werden kann und in welcher Form dies eine Verletzung ihrer Selbstachtung darstellt. Dabei werde ich nicht auf die juristische Handhabe der Genozidleugnung im Rahmen einer Rassismusstrafnorm eingehen ${ }^{30}$, bzw. wie die Genozidleugnung im Verhältnis zur Meinungsäusserungsfreiheit steht. ${ }^{31}$ Vielmehr möchte ich einen versteckten Aspekt der Beziehung zwischen Genozidleugnung und Selbstachtung der Nachfahren beleuchten, wonach Genozidleugnung nicht als Form der Hassrede interpretiert, sondern vielmehr aufgezeigt werden sollte, wie gesellschaftliche Vorurteile anderen ungerechtfertigterweise die Glaubwürdigkeit und letztlich zentrale menschliche Fähigkeit aberkennen, zuverlässiges Wissen zu generieren und weiterzugeben.

\footnotetext{
${ }^{29}$ Siehe Fricker 2007

${ }^{30}$ Vgl. beispielsweise Art. 261bis des Schweizerischen Strafgesetzbuches: https://www.admin.ch/opc/de/classified-compilation/19370083/index.html\#a261bis

${ }^{31}$ Die Diskussion eines solchen Falles findet sich im Beitrag von Sévane Garibian in diesem Bd.
} 


\section{Genozidleugnung als epistemische Ungerechtigkeit}

Miranda Fricker versteht unter einer epistemischen Ungerechtigkeit ein sowohl epistemisches als auch ethisches Fehlverhalten innerhalb unserer alltäglichen, sozialen Praxis des Wissensaustauschs. Es geht hier also weniger um die epistemische Frage danach, unter welchen Bedingungen eine Überzeugung gerechtfertigt oder wahr ist, sondern inwiefern andere unsere Fähigkeit zur Überzeugungs- und Wissensgenerierung grundsätzlich und ungerechtfertigterweise in Abrede stellen können und uns dadurch als epistemische Akteure zurückweisen. Dadurch muss jedoch zunächst anerkannt werden, dass die Praxis des Wissens eine solche soziale Dimension hat. Fricker identifizert nun eine Ungerechtigkeit in Bezug auf Zeugenschaft - die sogenannte testimonial injustice - welche vorliegt, wenn einem wissenden Subjekt aufgrund eines Vorurteils gegenüber ihrer sozialen Identität nicht die Glaubwürdigkeit entgegengebracht wird, die sie ansonsten erhalten würde. Es handelt sich bei einer epistemischen Ungerechtigkeit also nicht schlicht um ein ungewolltes Missgeschick oder einen naiven Fehler. Dabei ist insbesondere im Hinblick auf Frickers These wichtig, zwischen Stereotypen und Vorurteilen zu unterscheiden. Gemäß Fricker sind Stereotypen nicht grundsätzlich schädlich. Sie sind zunächst nur weitverbreitete Ansichten darüber, dass bestimmte Eigenschaften für Personen oder eine Gruppe charakteristisch sind. Dies können durchaus mehr oder weniger zuverlässige, positive wie auch negative Eigenschaften sein. ${ }^{32}$ Auch in der Diskussion um die Entstehung und das Selbstverständnis identitätsstiftender Gruppen, wie sie bei Margalit genannt werden, kommt man nicht umhin, mit Stereotypen zu arbeiten. Dies insbesondere, weil sie in alltäglichen Begegnungen auch nützlich sein können, denn soziale Identitäten tragen spezifische Erwartungen oder Annahmen beispielsweise darüber mit, wie eine Person behandelt werden soll oder behandelt werden möchte. Um ausserdem überhaupt von einer identitätsstiftenden Gruppe sprechen zu können, muss diese Gruppe öffentlich erkennbar sein. Oder, wie es Margalit und Raz nennen, „a highly visible social profile“ haben. ${ }^{33}$ Diese Macht der Identität („,identity power“ nach Fricker) ist jedoch auch ein integraler Bestandteil der Mechanismen von Wissensaustausch, denn Zuhörende brauchen

\footnotetext{
${ }^{32}$ Durchaus können unter gewissen Umständen bereits Stereotypen als moralisch fragwürdig erachtet werden, da man dadurch die Gefahr läuft, grundsätzlich heterogene Gruppen zu homogenisieren und ihnen essentielle Eigenschaften zuzuschreiben. Mit andern Worten kann eine bestimmte Beschreibung einer Gruppe dazu führen, dass gewisse Mitglieder dieser Gruppe sich unter dieser Beschreibung nicht repräsentiert fühlen. Der Einfachheit halber werde ich aber diese Unterscheidung, auf die Fricker besteht, beibehalten, da mir zumindest gemäss ihrem Verständnis von Vorurteilen diese als ethisch und epistemisch gravierender erscheinen als Stereotypen, die auch harmlos oder pragmatisch relevant sein können. In Bezug auf Letzteres sei kurz auf das von Gayatri Spivak begründete Konzept des „strategic essentialism“ verwiesen, welches besagt, dass marginalisierte soziale Gruppen trotz ihrer Heterogenität diese kurzzeitig beiseitestellen können, um einen Sinn kollektiver Identität zu formen und sich dadurch für ein bestimmtes Ziel politisch Gehör zu verschaffen. Vgl. Spivak 1987, S. 205

${ }^{33}$ Margalit und Raz 1990, S. 446
} 
soziale Stereotypen als Heuristiken, um spontan die Glaubwürdigkeit ihres Gesprächspartners einschätzen zu können. Dieser Gebrauch von Stereotypen kann angebracht und richtig sein, er kann jedoch auch irreführend sein, je nachdem, um welche Art Stereotyp es sich handelt. ${ }^{34}$ Aus Stereotypen werden nämlich dann Vorurteile, wenn diese selbst angesichts widersprechender empirischer Fakten oder Gegenbeispielen aufrechterhalten werden. Vorurteile sind daher, im Gegensatz zu Stereotypen, irrationale und hartnäckige, ja falsche Urteile, die trotz Gegenbeispielen aufrechterhalten werden. Sie sind, in Frickers Worten, “judgments, which may have a positive or a negative valence, and which display some (typically, epistemically culpable) resistance to counter-evidence owing to some affective investment on the part of the subject”. ${ }^{35}$ Dabei sind für unsere Zwecke insbesondere jene Vorurteile ethisch problematisch, die durch ein negatives „affective investment“ charakterisiert sind. Das heisst, sie sind mit einer abwertenden Haltung gegenüber der sozialen Identität einer Person verbunden. Für Fricker begeht der Zuhörer hier also zweierlei Fehler:

[T]here is an epistemic dysfunction in the exchange - the hearer makes an unduly deflated judgment of the speaker's credibility, perhaps missing out on knowledge as a result; and the hearer does something ethically bad - the speaker is wrongfully undermined in her capacity as a knower. ${ }^{36}$

Damit erfasst sie also sowohl eine epistemische als auch ethische Ungereimtheit innerhalb der Praxis des Wissensaustauschs. Nun soll gezeigt werden, inwiefern ein Fall von institutioneller Genozidleugnung im Lichte einer solchen vorurteilshaften, ethisch wie auch epistemisch verwerflichen Glaubwürdigkeits-,„Ökonomie“ untersucht werden kann. Dadurch wird auch ersichtlich, weshalb die Genozidleugnung für Nachfahren eine andere Bedeutung hat als für andere epistemische Akteure wie AkademikerInnen, die nicht der entsprechenden sozialen Gruppe angehören. Natürlich werden beispielsweise auch HistorikerInnen in ihrer Glaubwürdigkeit ungerechtfertigterweise unterminiert, jedoch mit Verweis darauf, dass sie sich beispielsweise auf Quellen oder Archive stützen, die nicht glaubwürdig sind, da sie von ArmenierInnen stammen oder „blosses Propagandamaterial“ sind. Im Hintergrund steht die Vorannahme, dass jeder Begründungsversuch des Genozids nur schon deshalb falsch ist, da es der „Sache der Armenier“ dient, gekoppelt mit ihrem Bedürfnis, „Hass gegenüber Türken“ zu säen. ${ }^{37}$

\footnotetext{
${ }^{34}$ Vgl. Fricker 2007, S. 16f.

${ }^{35}$ Fricker 2007, S. 35

${ }^{36}$ Ebd., S. 17

${ }^{37}$ Die Behauptung, dass die Anerkennung des Genozids eine Hassbekundung gegenüber TürkInnen sei, scheint an Absurdität kaum zu überbieten. Sie findet sich aber bis heute in der offiziellen Rhetorik der Türkei, indem jegliche Versuche der Völkermordanerkennung als islamophob oder turkophob bezeichnet werden. Siehe z.B. die Presseerklärung der Türkei vom 2. Juni 2016, nachdem die deutsche Regierung die Völkermordresolution
} 
Vorurteile sind also gekennzeichnet durch eine gewisse Blindheit oder einen affektiv motivierten Widerstand gegenüber Gegenbeweisen. Dies hiesse, dass es Genozidleugnern gerade nicht um die Rechtfertigung von Wissen oder „die Wahrheit“ geht, sondern dass sie andere Motive verfolgen. Gerade die Beweisfrage ist es, welche im Kontext des Genozids an den ArmenierInnen und dessen Leugnung stets für besondere Diskussion sorgt. Mit andern Worten: Der unaufhörliche Ruf nach „Faktizität“ und „Objektivität“ der historischen oder jedweder wissenschaftlichen Untersuchung und der Quellen, auf die sie sich stützt. So weisen Alexandra Garbarini und Boris Adjemian darauf hin, dass gerade deshalb der Fokus in der wissenschaftlichen Aufarbeitung des Genozids bisher auf offiziellen Dokumenten und diplomatischen oder regierungsamtlichen Quellen lag, sowie auf Zeugenaussagen von Drittparteien, wie beispielsweise MissionarInnen, ÄrztInnen oder ausländischen Konsularbeamten, die sich während des ersten Weltkriegs in Anatolien befanden. Erst Anfang der 90er Jahre, so Garbarini und Adjemian, begann man auch die Zeugenaussagen der Opfer und Überlebenden des Genozids als wichtige wissenschaftliche Quellen zu berücksichtigen. ${ }^{38}$ Diese verzögerte Würdigung erklären sie teilweise durch den Verweis auf sprachliche Verständnis- bzw. Zugangsprobleme sowie die Tatsache, dass sie bloß als Manuskripte in den Archiven vorhanden waren. Doch der prekäre Status der Opferaussagen wurde durch die langjährige Genozidleugnung nochmals verschlimmert, wodurch die Geschichtswissenschaften sozusagen gefangen waren in der unaufhörlichen Aufforderung, die Fakten zu beweisen insbesondere das Element der expliziten Zerstörungsabsicht - während zugleich die Aussagen der Überlebenden, selbst jene, die unmittelbar nach den Ereignissen aufgenommen wurden, diese Ereignisse nicht mit genügender Präzision zu repräsentieren schienen. Allerdings haben es im Kontext der Genozidleugnung auch solche offiziellen Dokumente teilweise schwer, als Beweismaterial akzeptiert zu werden. So werden gerade auch Quellen aus britischen oder französischen Archiven als unglaubwürdiges, nicht ernst zu nehmendes Propagandamaterial wahrgenommen. Ausserdem weist Garbarini in einem weiteren Essay auf die Paradoxie hin, den Aussagen und Berichten von Drittparteien mehr Unabhängigkeit und Objektivität zuzuschreiben als den direkt betroffenen Opfern. Sie diskutiert dies anhand Viscount James Bryce und Arnold J. Toynbees The Treatment of Armenians in the Ottoman Empire 1915-1916:

On the one hand, incorporating victim testimony in the composition of [this document volume] proved essential in order to inform, convince, and awaken sympathy among readers. On the other hand, the editors assumed that they would be unable to overcome

\footnotetext{
verabschiedet hat: http://www.mfa.gov.tr/no_-125_-2-june-2016_-press-release-regarding-the-resolution-by-theparliament-of-the-federal-republic-of-germany-of-2-june-2016-on-the-events-of-1915.en.mfa

${ }^{38}$ Vgl. Garbarini und Adjemian 2015, S. 18
} 
their readers' doubt and mistrust, their refusal even, to accept this type of source as valid and believable documentation. ${ }^{39}$

Es geht hier also konkret um die Frage der Glaubwürdigkeit der ZeugInnen. Auch hier lässt sich beobachten, dass im Hinblick auf die Glaubwürdigkeit der tatsächlichen Opfer ein Vorurteil mitspielt, nämlich, dass direkt Betroffene eines Verbrechens qua Opferstatus nicht zuverlässig, sprich „objektiv“ über ihre Erfahrungen berichten, sondern diese zugunsten anderer Interessen verzerren würden. Dies mache scheinbar die Beisetzung „neutraler” Quellen nötig, um die behauptete Unzuverlässigkeit der Opferaussagen auszugleichen:

In elevating the evidence issuing from witnesses "who belong to neutral or belligerent countries,” i.e., witnesses of primarily American, German, and Swiss national origin, Bryce implied that the Armenian victims were not the primary "moral witnesses" or "bearers of testimony". The fact that they had experienced violence directly did not qualify them to be the privileged communicators of their experiences. On the contrary, their personal experiences rendered their testimony suspect because it made them more likely to fabricate stories in order to advance their political, emotional, or financial interests. ${ }^{40}$

Die Untersuchung von Garbarini zeigt ein essentielles Problem auf, nämlich unsere paradoxe Herangehensweise an angeblich objektive, neutrale oder unbefangene Quellen und die damit unmissverständlich zusammenhängenden Vorurteile, die zwar nicht in „böser Absicht“ erfolgen - denn es wird ja versucht, anhand von glaubwürdigen „neutralen“ Aussagen die Aussagen der Opfer glaubwürdig zu machen - jedoch unmissverständlich ein grobes Vorurteil gegenüber den Opfern darstellt. Es handelt sich hierbei also um ein paradigmatisches Beispiel für eine testimonial injustice. Die Scheinheiligkeit dieser unterschiedlichen epistemischen Beurteilung der ZeugInnen möchte ich mit einem letzten wichtigen Zitat aus Garbarinis Essay nochmals beleuchten, wo sie ebenso auf die jeweilige Befangenheit von „neutralen“ Zeugen hinweist:

Over and over again in their accounts of Armenian atrocities, missionaries, doctors, nurses, teachers, even consuls, describe the dangers they faced and the vulnerability they felt in their dealings with Turkish officials. [...] some among them also suffered the loss of members of their parties, including close friends, spouses, and siblings. And perhaps most agonizing of all, they witnessed the deportations and deaths of Armenians who were their students, patients, colleagues, and friends [...]. In their eyewitness accounts, they detail their feelings of terror, sorrow, helplessness, and anger, including avowals of their disgust for Turks and for Muslims in general. Yet, no acknowledgement exists in Toynbee and Bryce's editorial framing of this document collection of the suffering and emotional investments of so-called "neutral" eyewitnesses. It is as if their individual

\footnotetext{
${ }^{39}$ Garbarini 2015, S. 120

${ }^{40}$ Garbarini 2015, S. 125, teilw. zit. n. Greenspan et al. 2014, S. 191
} 
experiences had no bearing and only their political/national/ethnic/religious group belonging informed the writing and the reading of their testimonies. ${ }^{41}$

Bestimmte Formen der türkischen Genozidleugnung sind ein Paradebeispiel für dieses paradoxale Vermischen von Ansprüchen an professionelle, wissenschaftliche Methoden und Standards (Stichworte „Neutralität“, „Objektivität“, etc.) mit irrationalen Motiven, respektive Vorurteilen im Hinblick auf die Auswahl der Wissens- und Beweisquellen. Unter den generellen Mustern der mittlerweile professionalisierten und „verwissenschaftlichten“ Leugnung des Genozids an den ArmenierInnen identifiziert Maria Karlsson beispielsweise zum einen (i) Rationalisierung und Trivialisierung durch das Bestreiten von verfügbaren stichhaltigen Beweisen ${ }^{42}$, sowie (ii) ein bestimmtes Selbstverständnis, respektive Selbsttäuschung durch Selbstbestätigung bei gleichzeitiger Herabsetzung anderer ${ }^{43}$. Diese zwei Muster sind hier insofern miteinander verbunden, als die Selbstwahrnehmung und die Wahrnehmung des „Anderen“ auch die Einstellung gegenüber Beweisen mitbeeinflusst. Während die Strategie der (i) Rationalisierung und Trivialisierung des Genozids nicht unbedingt bestreitet, dass etwas passiert ist, geht Rationalisierung beispielsweise so weit, zu behaupten, dass es nicht genügend stichhaltige Beweise gibt, um daraus den Schluss eines Genozids zu ziehen. Argumente der Trivialisierung behaupten wiederum, dass die Opferzahl zu niedrig ist, um von Genozid zu sprechen oder dass selbst angesichts „vieler Armeniertode“ keine Absicht vorhanden war, diese zu töten. Im Gegensatz zur absoluten Leugnung, dass etwas passiert sei, weisen Argumente der Rationalisierung und Trivialisierung einen weit höheren Grad an historischen Details auf, wobei Archivmaterial diskutiert und in ein kohärentes Narrativ gesetzt wird. Gemäss Karlsson sind diese Argumente "often made under the guise of legitimate scholarly debate, and half-truths are presented as objective evidence and solid interpretations". ${ }^{44}$

Die Unaufrichtigkeit erweist sich jedoch nicht nur in der selektiven Auswahl von Archivmaterial - was für sich genommen methodologisch sinnvoll sein kann - sondern in der gleichzeitigen Ablehnung spezifischer, offensichtlich widersprechender Beweise, die oftmals verstrickt ist mit Vorurteilen oder Verschwörungstheorien über deren Entstehung. Als Beispiel hierfür nennt Karlsson Heath Lowrys Ablehnung der Zeugenaussagen und Memoiren von Henry Morgenthau, dem amerikanischen Botschafter im Osmanischen Reich von 1913-1916, den er als blossen Propagandisten abtut:

\footnotetext{
${ }^{41}$ Garbarini 2015, S. 127, zit. n. Soulahian Kuyumjian 2010, S. 85 sowie Sarafian 2000

${ }^{42}$ Karlsson 2010, S. 50ff.

${ }^{43}$ Ebd., S. 65ff.

${ }^{44}$ Ebd., S. 41
} 
In order to disqualify the bystander memoir, and in effect the Armenian Genocide as a whole, Lowry spotlights Morgenthau's Armenian connections, maintains that the memoirs in fact were written by someone else, and determines that the sole purpose of writing the memoirs was a 'short-term propaganda coup'. ${ }^{45}$

Hier zielt die Strategie der Verschwörungstheorie darauf ab, die Glaubwürdigkeit von Beweismaterial zu erschüttern. Dabei wird der Genozid gerne als Mythos bezeichnet, welcher zu Propagandazwecken während des Kriegs von Seiten der Alliierten kreiert wurde, um im Westen die öffentliche Meinung gegen die Türkei und Deutschland zu mobilisieren. Die ArmenierInnen, so die Behauptung, hätten die „Christian versus Muslim ethnic-card“ ausgespielt und Geschichten über ein erfundenes Massaker erzählt, um seitens christlicher Nationen Sympathien für ihre revolutionären Vorhaben zu wecken oder gar Kapital daraus zu schlagen. ${ }^{46}$ Solche Behauptungen dienen ausserdem dazu, die Opfergruppe als unmoralisch und opportunistisch auszuweisen; offenbar bereit, eine furchtbare Lüge dazu zu benützen, um an Geld zu kommen. Dass bei diesem Muster die Ideologie über die Logik herrscht, zeigt sich auch an der Tatsache, dass die behauptete Unzuverlässigkeit eines Autors oder einer Quelle zum Anlass genommen wird, den Genozid insgesamt als Lüge zu bezeichnen.

Nebst dieser Strategie, die „anderen“ als unzuverlässige Beweisquellen zu erachten, ist die Art und Weise, wie sich die Leugner selbst wahrnehmen. Damit kommen wir zum zweiten bereits erwähnten Leugnungsmuster der (ii) Selbstbestätigung und Herabsetzung anderer. Unter den von Karlsson untersuchten Werken von Genozidleugnern ${ }^{47}$ stellt sie insbesondere deren Bedürfnis fest, sich als objektive Wahrheitssuchende zu porträtieren. ${ }^{48}$ Was den Leugnern jedoch wichtig ist, ist nicht die Wahrheit an sich, sondern der Anschein von Wahrheit, Glaubwürdigkeit und Objektivität: "In order to create this appearance of truth and professionalism, deniers both tend to confirm their own excellence, and constantly point out the inadequacies of 'traditional' scholars." ${ }^{49}$ Dieses an und für sich noble wissenschaftliche Ziel ist im Grunde aber eine selbsttäuschende und unprofessionelle Art und Weise, sich selbst zum "Märtyrer der Geschichtswissenschaft" zu erheben. ${ }^{50}$ Das Unprofessionelle ist daran zu erkennen, dass diese Selbstbestätigung meist mit der persönlichen Herabsetzung anderer einhergeht. Willkommene Zielobjekte sind dabei insbesondere armenische WissenschaftlerInnen, aber auch alle, die die sogenannten "traditionelle Ansicht" des Genozids vertreten, respektive „armenierfreundliche WissenschaftlerInnen“, die - geblendet von den

\footnotetext{
${ }^{45}$ Ebd., S. 53, zit. n. Lowry 1990, S. 11, 14ff., 23 und 37

46 Karlsson 2010, S. 59, zit. n. Weems 2002, S. 115

47 Namentlich Bernard Lewis, Samuel Weems, Stanford Shaw, Heath Lowry und Justin McCarthy

48 Karlsson 2010, S. 65

${ }^{49}$ Ebd., S. 66

${ }^{50}$ Ebd., S. 67
} 
ArmenierInnen - bloss über eine sehr eingeschränkte Sicht der Dinge verfügen und diesen Standpunkt verteidigen würden. ${ }^{51}$ Die Ironie liegt dabei gerade darin, dass Genozidleugner ihre Gegner für jene Dinge anklagen, für die sie selbst angeklagt werden. Welche Argumente auch immer hervorgebracht werden, um Genozidleugner zu enttarnen, dieselben Argumente werden ihrerseits verwendet, um die Vertreter der „traditionellen Genozidthese“ zu diskreditieren. Für diejenigen, die nicht viel über das Thema wissen oder nicht imstande sind, selbst Nachforschungen $\mathrm{zu}$ betreiben, ist dies enorm verwirrend und macht mit Sicherheit den Eindruck, als ob der Genozid eine historisch bestreitbare Tatsache wäre.

Ziel dieses Abschnitts war es, einige Muster aufzuzeigen, wie Genozidleugner mit Beweismaterial umgehen oder deren Glaubwürdigkeit hinterfragen. Die epistemische Integrität, respektive die Aufrichtigkeit und Kompetenz anderer zu hinterfragen ist dabei eine verbreitete Strategie. An sich nicht unbedingt verwerflich, ist diese Strategie im Kontext der Genozidleugnung gerade deshalb verwerflich, da sie auf ungerechtfertigten Vorannahmen beruht. Der Genozid an den ArmenierInnen wird von vornherein als politischer Mythos erachtet, dem es jedweder empirischen Grundlage fehlt. Beweismaterial wird dadurch nicht auf der Grundlage guter Gründe zurückgewiesen oder durch berechtigte Quellenkritik - selbst wenn dies manchmal den Anschein haben mag - sondern aufgrund unhaltbarer Vorurteile, die aus persönlichen oder politischen Gründen aufrechterhalten werden. In einem letzten Schritt soll nun gezeigt werden, was es bedeutet, Personen in ihrer Fähigkeit der Wissensweitergabe zu verletzen, respektive ihre epistemische Kompetenz ungerechtfertigterweise in Abrede zu stellen und sie dadurch erneut zu demütigen.

\section{Epistemisches Selbstvertrauen und Selbstachtung}

Die Annahme, dass andere ungerechtfertigterweise unsere epistemischen Kompetenzen untergraben können, setzt voraus, dass es sich bei der Entstehung von Wissen und dem Formen unserer Überzeugungen auch um eine sozial situierte Praxis handelt. Wissen wird nicht nur nach inhaltlichen Kriterien beurteilt. Vielmehr werden im Rahmen des Wissensaustauschs auch die Subjekte beurteilt, die einen Wissensanspruch stellen oder Wissen weitergeben möchten, und zwar im Hinblick auf ihre Kompetenz und Aufrichtigkeit. ${ }^{52}$ Laut Fricker ist eine testimonial

\footnotetext{
${ }^{51}$ Ebd., zit. n. Lowry 1990, vi. Hierzu sei angemerkt, dass eine „weitere“ Sicht der Dinge, die der Komplexität der historischen Gegebenheiten gerecht würde, vielleicht etwas zur Erklärung beitragen würde, wie und weshalb es zum Genozid kam - doch an der Bewertung als Genozid würde dies nichts ändern, geschweige denn die Verbrechen rechtfertigen.

${ }^{52}$ Für Fricker sind dies die beiden Komponenten, die epistemische Vertrauenswürdigkeit begründen. Vgl. Fricker 2007, S. 45
} 
injustice besonders dann ernsthaft schädigend, wenn sie fortdauernd und systematisch ist, wobei schließlich das Subjekt in seiner Fähigkeit der Wissensweitergabe (und der Wissensgenerierung selbst) untergraben wird. Da dies eine essentielle menschliche, mit unserem Vernunftvermögen verbundene Fähigkeit ist, kann hier auch von einer intrinsischen Ungerechtigkeit gesprochen werden. ${ }^{53}$ Die Beziehung zwischen einer testimonial injustice und der Selbstachtung einer Person kann in Frickers Worten wie folgt beschrieben werden:

The fact that the primary injustice involves insult to someone in respect of a capacity essential to human value lends even its least harmful instances a symbolic power that adds a layer of harm of its own: the epistemic wrong bears a social meaning to the effect that the subject is less than fully human. When someone suffers a testimonial injustice, they are degraded qua knower, and they are symbolically degraded qua human. ${ }^{54}$

Durch eine epistemische Ungerechtigkeit wird ein Mensch demnach nicht voll als Mensch geachtet. Wie jedes Vorurteil, welches eine negative Bewertung einer sozialen Gruppe im Hinblick auf zentrale menschliche Eigenschaften beinhaltet, kann auch eine testimonial injustice als Fall von Demütigung verstanden werden.

Unsere Fähigkeit zur Wissensgenerierung ${ }^{55}$ und -weitergabe ist nicht nur abhängig von äußeren Kräften. Als verantwortliche epistemische Akteure sollten wir über eine grundlegende Zuversicht in unsere epistemischen Fähigkeiten und Kompetenzen verfügen und uns somit auch bis zu einem gewissen Masse als Autorität anerkennen, selbst zu entscheiden, anhand welcher Gründe wir unser Wissen rechtfertigen oder revidieren. Wir sollten also zunächst genügend Selbstvertrauen entwickeln im Hinblick auf unsere Fähigkeit, durch Reflexion zu „wahrem Wissen“ und entsprechenden Überzeugungen zu gelangen. ${ }^{56}$ Dabei ist nicht jeder wirksame Einfluss auf unser Wissen eine Unterminierung unseres epistemischen bzw. intellektuellen Selbstvertrauens und somit ethisch verwerflich: Im Prinzip sollte Wissen stets zugänglich sein für kritische Hinterfragung. Wir könnten jederzeit mit Gründen konfrontiert werden, die unsere Überzeugungen möglicherweise auf den Kopf stellen. Als verantwortungsvolle epistemische Akteure sollten wir schliesslich auch eine angemessene Offenheit gegenüber Gründen, respektive den Worten anderer entwickeln. Doch wenn es um die Entscheidung darüber geht, auf welcher Grundlage wir andere Personen im Hinblick auf ihre Glaubwürdigkeit beurteilen, sind wir auch im Bereich der Ethik. Hier kann wiederholte und systematische epistemische

\footnotetext{
${ }^{53}$ Gemäß Martha Nussbaum lassen sich solche essentiellen (im Unterschied zu akzidentiellen) menschlichen Eigenschaften feststellen durch einen sogenannten „internalistischen Essentialismus“, worunter sie einen historisch fundierten empirischen, von der menschlichen Erfahrung abgeleiteten Essentialismus versteht. Vgl. Nussbaum 1992, S. 208

${ }^{54}$ Fricker 2007, S. 44

${ }^{55}$ Als mögliche Quellen von Überzeugungen, Wissen und Wahrheit gelten Wahrnehmung, Gedächtnis, Bewusstsein, Vernunft und Zeugenschaft (als sozial begründetes Wissen). Vgl. Audi 2011, S. 13ff.

${ }^{56}$ Vgl. Jones 2012
} 
Ungerechtigkeit zu einer Form von Unterdrückung werden. Dabei spricht man nicht mehr nur von einem Fall des Verlusts von Selbstvertrauen, sondern dies tangiert auch die Selbstachtung der Betroffenen. Durch die andauernde institutionelle Leugnung des Genozids wird ArmenierInnen nicht nur der Zugang zu Wissen verwehrt, welches relevant ist für ihre praktische Identität. Nebst diesem Kontrollverlust über ihre praktische Identität werden ihnen zentrale menschliche Fähigkeiten aberkannt, sie werden nicht als vollwertige epistemische Subjekte behandelt, was ihnen einen objektiven normativen Grund gibt, sich als gedemütigt zu erachten.

\section{Einwände und Schlussbemerkungen}

Im Zusammenhang mit der offiziellen türkischen Leugnung des Armeniergenozids wird immer wieder hinterfragt, ob denn dieses Beharren auf dem Wort „Genozid“ notwendig ist, insbesondere angesichts der umstrittenen völkerrechtlichen Definition. Reicht nicht die Anerkennung der Gräuel als Massaker und Vertreibungen, egal aus welcher Absicht diese begangen wurden? In diesem Beitrag habe ich versucht zu zeigen, dass trotz der Mängel der UN Konvention das Verbrechen des Genozids auf ein spezifisches Verbrechen gegen die Menschlichkeit, respektive eine spezifische menschliche Grausamkeit hinweist, nämlich die Demütigung im Sinne eines Ausschlusses einer moralisch legitimen identitätsstiftenden, i.e. sozialen Gruppe nicht nur aus der politischen, sondern letztlich aus der Menschengemeinschaft. Der Begriff „Genozid“ trägt daher eine spezifische moralische Bedeutung mit sich und legt dadurch den Rahmen der Art und Weise fest, wie wir über diese Geschehnisse diskutieren und darauf reagieren sollen. Dabei lassen sich die Unrechtserfahrungen der ArmenierInnen nicht angemessen fassen durch Bezeichnungen wie „Bürgerkrieg“, „Umsiedlung“ oder „Wirren des Kriegs“. ${ }^{57}$ Doch die offizielle Leugnung historischer Tatsachen, genauer genommen ihrer angebrachten Interpretation und Bewertung, stellt nicht nur eine ungerechte Behandlung der Nachfahren der Opfer dar. Eine kontinuierliche Lüge hält auch andere Rezipienten, respektive andere Mitglieder der Gesellschaft davon ab, ihre Entscheidungen anhand genügend angemessener Informationen zu treffen. Sie führt zu einem generellen Wissensverlust, wodurch auch andern Mitgliedern der Gesellschaft wichtiges Wissen verwehrt wird. Dadurch werden sie

\footnotetext{
${ }^{57}$ Vgl. Schrodt 2014, S. 311ff. Schrodt weist hier darauf hin, dass all diese relativierenden Bezeichnungen der Geschehnisse das Versagen oder eben sogar die Weigerung der Osmanischen Regierung, die regionalen Spannungen zu deeskalieren, missachten. Gerade das Gegenteil war nämlich der Fall: “[...] [The Ottoman state] even determinedly affirmed resentment and prejudice by shedding collective suspicion on their Ottoman Armenian subjects, and pursued a proactive policy of repressions.” (Ebd.)
} 
Opfer einer „fremdverschuldeten Ignoranz“ im Hinblick auf Wissen, welches für das friedliche gesellschaftliche Zusammenleben zentral ist. ${ }^{58}$

Ein weiterer Einwand lautet, dass die Erinnerung an ein Verbrechen, dem die gesellschaftliche Spaltung anhand ethnischer oder religiöser Zugehörigkeit inhärent ist, diese Division bloß tradieren und somit auch den „gegenseitigen Hass" aufrechterhalten würde, anstatt dass man sich quasi „einer einzigen Menschengemeinschaft zugehörig“ erachtet und gemeinsam vorwärtsschaut. Diese Sichtweise ist gefährlich und mag vielleicht in einer idealen Welt funktionieren. Zunächst sei vorangestellt, dass das Recht einer Kultur, ihre Selbstachtung wiederherzustellen oder sich wiederzubehaupten, nicht impliziert, einem blinden Konservatismus anheim zu fallen. Einer moralisch legitimen identitätsstiftenden Gruppe zu erlauben, ihre Legitimität und insbesondere ihre legitime Zugehörigkeit zur politischen Gemeinschaft wieder zur Geltung zu bringen ist nicht gleichbedeutend mit der Proklamation von kulturellen Praktiken ohne stetige Reflektion über deren Legitimität und Zweck. Dabei ist das Verbrechen des Genozids ein Verbrechen, welches das grundsätzliche Existenzrecht dieser Gruppe und damit auch die Möglichkeit zur Veränderung in Abrede stellt. Beim Genozid geht es schließlich nicht um eine ausgefeilte Kritik an einer bestimmten Form oder Lebensweise der Gruppe, denn die Genozidtäter sind keine sozialanthropologischen Experten - auch wenn es die entsprechenden Ideologen gerne vorgeben, zu sein. Vielmehr spricht ein Genozid der Gruppe auch die grundsätzliche Möglichkeit ab, sich zu entwickeln und zu verändern. Diese Überlegungen sind wichtig, denn angesichts der kosmopolitischen und postmodernen Ideologien der Grenzenlosigkeit können es Anliegen von Minderheitengruppen schwierig haben, ihre legitimen Ansprüche an ihr Existenzrecht geltend zu machen. Die Idee eines kosmopolitischen Menschen mag als Ideal erstrebenswert sein. Dieses jedoch gegen Anliegen von kulturellen Gruppen aufzuwiegen heisst, in unserer nicht-idealen Welt mehr Leid zu verursachen, oder vorhandenes Leid nicht zu lindern. Es handelt sich bei meiner Auseinandersetzung also um eine Ethik für nicht-ideale Welten, was das Anstreben und die Beförderung kosmopolitischer Werte sowie die Dekonstruktion sozialer Identitäten nicht ausschliesst, jedoch dies auch nicht aufgezwungen werden sollte. Denn auch dieses Ideal - wie im Übrigen auch das Ideal des „Friedens“ - kann zu politischen Zwecken missbraucht werden,

\footnotetext{
${ }^{58}$ Die Tatsache, dass in den offiziellen Geschichtsbüchern der Türkei weiterhin die ArmenierInnen als Vaterlandsverräter porträtiert werden, die die nationale Sicherheit bedrohen und Türken ermordet haben, kombiniert mit der selbstgerechten Beschreibung der Gegenmassnahmen u.a. als „Umsiedlungen“ führt in manchen, nationalistisch gesinnten türkischen Kreisen bis heute zu Ressentiments gegenüber den ArmenierInnen und einem grundlegenden Unverständnis gegenüber ihrer Forderung nach Genozidanerkennung. Vgl. Taner Akçam, "Textbooks and the Armenian Genocide in Turkey: Heading towards 2015" http://armenianweekly.com/2014/12/04/textbooks/. [Zugegriffen: 18. April 2016]
} 
um ethisch berechtigte Minderheitenanliegen politisch nicht berücksichtigen zu müssen. Schliesslich sei darauf hingewiesen, dass sofern das Erinnern an Genozid in manchen Fällen tatsächlich zur Tradierung von Hass und sozialen Konflikten führt, dies eher als ein Zeichen bereits fehlgeschlagener oder fehlgeleiteter Aufarbeitung gedeutet werden kann, als dass diese Eigenschaften als der Erinnerung inhärent erachtet werden sollten.

\section{Literaturverzeichnis}

Anderson, Elizabeth. 1993. Value in Ethics and Economics. Cambridge, Mass.: Harvard University Press.

Audi, Robert. 2011. Epistemology: A Contemporary Introduction to the Theory of Knowledge. 3. Aufl. New York: Routledge.

Bauer, Yehuda. 2009. Genocide Prevention in Historical Perspective. Politorbis 47: 25-32.

Blustein, Jeffrey M. 2014. Forgiveness and Remembrance: Remembering Wrongdoing in Personal and Public Life. New York: Oxford University Press.

Brendel, Elke. 2013. Wissen. In Grundthemen Philosophie, hrsg. Dieter Birnbacher, Pirmin Stekeler-Weithofer, Holm Tetens. Berlin: Walter de Gruyter GmbH \& Co.

Campbell, Sue. 2003. Relational Remembering: Rethinking the Memory Wars. Lanham, MD: Rowman \& Littlefield Publishers, Inc.

Chalk, Frank. 1994. Redefining Genocide. In Genocide: Conceptual and Historical Dimensions, hrsg. G. J. Andreopoulos, 47-63. Philadelphia: University of Pennsylvania Press.

Charny, Israel W. 1994. Towards a Generic Definition of Genocide. In Genocide: Conceptual and Historical Dimensions, hrsg. G. J. Andreopoulos, 64-94. Philadelphia: University of Pennsylvania Press.

De Greiff, Pablo. 2006. Justice and Reparations. In The Handbook of Reparations, hrsg. P. de Greiff, 451-77. New York: Oxford University Press.

Fricker, Miranda. 2007. Epistemic Injustice: Power and the Ethics of Knowing. Oxford: Oxford University Press.

Garbarini, Alexandra. 2015. Document Volumes and the Status of Victim Testimony in the Era of the First World War and Its Aftermath. Études arméniennes contemporaines 5: 113138.

Garbarini, Alexandra und Boris Adjemian. 2015. Mass Violence: From Experience to Knowledge. Études arméniennes contemporaines 5: 15-21

Greenspan, Henry, Sara R. Horowitz, Éva Kovács, Berel Lang, Dori Laub, Kenneth Waltzer and Annette Wieviorka. 2014. Engaging Survivors: Assessing "Testimony" and “Trauma” as Foundational Concepts. Dapim: Studies on the Holocaust, 28(3): 190-226.

Herman, Judith. 1992. Trauma and Recovery. New York: Basic Books.

Jones, Karen. 2012. The Politics of Intellectual Self-Trust. Social Epistemology, 26(2): 237251.

Karlsson, Maria. 2010. Tall Tales of Genocide: An Argumentative and Comparative Analysis of Western Denial of the Holocaust and of the Armenian Genocide. CFE Working Paper Series, 45. Lund University.

Kohler, Franziska. 2014. Institutionelle „Schubladisierung“ als Demütigung im Sinne Avishai

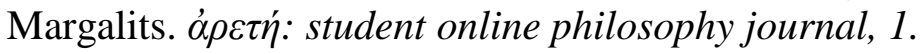

Lowry, Heath W. 1990. The Story behind Ambassador Morgenthau's Story. Istanbul: The Isis Press.

Margalit, Avishai. 2012. Politik der Würde. Cambridge, Mass.: Harvard University Press. 
Margalit, Avishai und Joseph Raz. 1990. National Self-Determination. The Journal of Philosophy, 87(9): 439-461.

May, Larry. 2010. Genocide: A Normative Account. New York: Cambridge University Press.

Nussbaum, Martha C. 1992. Human Functioning and Social Justice. In Defense of Aristotelian Essentialism. Political Theory 20: 202-246

Sarafian, A. (Hrsg.) 2000. Introduction. In The Treatment of Armenians in the Ottoman Empire, 1915-1916. Documents Presented to Viscount Frey of Fallodon by Viscount Bryce, Uncensored Edition. Princeton: Gomidas Institute.

Livingstone Smith, David. 2011. Less than Human: Why we Demean, Enslave, and Exterminate Others. New York: St. Martin's Press.

Schrodt, Nikolaus. 2014. Modern Turkey and the Armenian Genocide: An Argument about the Meaning of the Past. Cham: Springer International Publishing

Soulahian Kuyumjian, Rita. 2010. The Survivor: Biography of Aram Andonian. London: Gomidas Institute and Tekeyan Cultural Association.

Spivak, Gayatri Chakravorty. 1987. In Other Worlds: Essays in Cultural Politics. New York: Methuen.

Walker, Margaret Urban. 2006. Restorative Justice and Reparations. Journal of Social Philosophy, 37(3, Fall): 377-395.

Weems, Samuel A. 2002. Armenia: Secrets of a "Christian" Terrorist State: The Armenian Great Deception Series - Vol. 1. Dallas: St. John Press. 